$\underline{\text { Preprint typeset in JHEP style - HYPER VERSION }}$

$\mathrm{UCLA} / 04 / \mathrm{TEP} / 06$

UOSTP-04101

OU-HET 470

hep-th/0403249

\title{
Dilatonic Repulsons and Confinement via the AdS/CFT Correspondence
}

\author{
Dongsu Bak ${ }^{1}$, Michael Gutperle ${ }^{2}$, Shinji Hirano ${ }^{3}$ and Nobuyoshi Ohta ${ }^{4}$ \\ ${ }^{1}$ Physics Department, University of Seoul, Seoul 130-743, Korea \\ ${ }^{2}$ Department of Physics and Astronomy, UCLA, Los Angeles, CA 90095, USA \\ ${ }^{3}$ Department of Physics, Technion, Israel Institute of Technology, Haifa 32000, Israel \\ ${ }^{4}$ Department of Physics, Osaka University, Toyonaka, Osaka 560-0043, Japan
}

dsbak@mach.uos.ac.kr, gutperle@physics.ucla.edu,

hirano@physics.technion.ac.il, ohta@phys.sci.osaka-u.ac.jp

\begin{abstract}
We study a class of dilatonic deformations of asymptotically $\operatorname{AdS}_{5} \times S^{5}$ geometry analytically and numerically. The spacetime is non-supersymmetric and suffers from a naked singularity. We propose that the causality bound may serve as a criterion for such a geometry with a naked singularity to still make sense in the AdS/CFT correspondence. We show that the static string, the one corresponding to a large Wilson loop in the dual gauge theory, reveals confinement in a certain range of parameters of our solutions, where the singularity exhibits the repulsion that can well cloak the singularity from the static string probe. In particular, we find the exact expression for the tension of the QCD strings. We also discuss a possible interpretation of our solution in terms of unstable branes and their tachyon matter.
\end{abstract}




\section{Contents}

1. Introduction 1

2. Preliminary 4

3. Global Deformation 6

3.1 The causality bound 7

3.2 The repulson 8

3.3 The ADM mass 9

3.4 Repulson vs confinement 10

4. Deformation in the Poincaré Patch $\quad 12$

4.1 The causality bound 13

4.2 The repulson

4.3 The ADM mass 14

5. Nature of Singularity

6. Wilson Loop and Confinement $\quad 16$

6.1 Coulombic case 18

6.2 Confining case 19

6.3 The Mass Gap 21

7. Discussions 22

A. Hyperbolic Slicing 25

\section{Introduction}

The AdS/CFT correspondence [1], 2, 3] firmly established a duality between gravity and gauge theory, providing a remarkable realization of holography [4, 5]. There has been a plethora of applications and generalizations thus far, yet the non-supersymmetric cases have been less studied. A simple way to obtain the non-supersymmetric generalization is to add deformations that break supersymmetry. One of the simplest such deformations is to turn on only the dilaton. Such a dilatonic deformation on the one hand typically breaks all the 
supersymmetries and often suffers from a naked singularity. On the other hand it is simple and therefore quite tractable.

A non-singular dilatonic deformation, the "Janus" solution, was found in [6], which turns out to be dual to a non-supersymmetric dilation invariant deformation of $\mathcal{N}=4$ Supersymmetric Yang-Mills theory (SYM) by a certain exactly marginal operator. Even further the non-perturbative stability of the "Janus" solution was established against a broad class of fluctuation modes by a compelling argument developed in [7]. Thus the "Janus" solution was proven to be on a firm footing in the AdS/CFT correspondence, despite the lack of supersymmetry.

With this success, though moderate, it may be worthwhile to further explore possible dilatonic deformations of different characteristics. In fact there exists a wide class of simple dilatonic deformations (see, for example, [8, 9, 10]). It seems, however, rather rare that the dilatonic deformations lead to non-singular geometries. In general relativity a geometry with a naked singularity should be dismissed because of cosmic censorship. In string theory however a naked singularity can make sense if a brane interpretation is given, or a stringy resolution exists. In terms of the AdS/CFT correspondence, the problem of the singular geometry would be manifested by a causal violation of holography [11], - for the bulk/boundary correspondence to make sense, it is prohibited that any signal, sent from a point $\mathrm{P}$ on the boundary, reaches a distant point $\mathrm{Q}$ on the boundary, faster by traveling through the bulk than by traveling along the boundary. For instance, the negative mass AdS-Schwarzschild black hole is a typical example of geometry with a naked singularity. Indeed the causality bound of [1] excludes it from being a sensible geometry in the AdS/CFT correspondence.

In this paper, we take the causality bound as a postulate to diagnose which of singular geometries with asymptotically AdS space may make sense in string theory. The dilatonic deformations we consider in this paper all suffer from a (timelike) naked singularity. In fact adopting the causality bound rules out a certain range of parameter space of our solutions, but leaves a wide range to be sensible as gravity dual of the boundary gauge field theory. Physically the causality condition imposes the lower (non-negative) bound on the mass of our singular geometry. In other words, sufficiently energetic dilatonic deformations, even with the naked singularity, can clear the causality bound.

In particular three types of deformations are studied; the global patch, Poincaré patch, and hyperbolic slicing. In all cases, we have three independent parameters; $\phi_{\infty}$, the constant part of the dilaton, $k$, the strength of the nontrivial profile of the dilaton, and $A$ (or $\mu$ ), the energy of the geometry. The causality bound sets the lower (non-negative) bound on $A$ (or $\mu$ ) for any given $k$. In the case of the global patch, we carry out a numerical analysis to solve the condition for the causality bound and find the positive lower bound on $A$ for any given $k$. In the case of the Poincaré patch, the causality bound is somewhat trivial and 
analytically solved. It simply imposes $\mu$ to be non-negative. Our claim is that, within these parameter ranges, the AdS/CFT correspondence makes sense, and our geometry is dual to some (non-supersymmetric) states, labeled by $k$ and $A$ (or $\mu$ ), in $\mathcal{N}=4 \mathrm{SYM}$, where $k$ and $A$ (or $\mu$ ) are the expectation values of (supersymmetric completion of) $\operatorname{Tr} F^{2}$ and the energy $T_{00}$ respectively, following [12, 13].

There is yet another distinct range of parameters for our solutions. In this range, the naked singularity exhibits a strong repulsion overwhelming the attraction due to the negative cosmological constant. This can be regarded as an example of the repulson geometry [14, 15, 16]. A typical case of the repulson is again the negative mass black hole. We, however, find the overlap of the parameter ranges for the repulson and the causality. Thus the repulsion occurs even when the mass is positive in our case.

The repulson is, as we argue, a potential indication of confinement in the dual gauge theory. We demonstrate our claim in the case of the Poincaré patch, by calculating the static string, corresponding to the Wilson loop in the dual gauge theory [17, 18]. For a small loop, the static string is not pulled down deep enough to feel the deformation of geometry. Thus the quark-antiquark potential is shown to be Coulombic as in the AdS case. However, as the loop gets larger and larger, we find a (confining) scale far beyond which the quark-antiquark potential becomes linear. Unlike the AdS case, the long string would not be dragged far deep according to the simple IR/UV relation [19], but stops penetrating beyond the confining scale. In other words, the repulsion is strong enough to cloak the naked singularity from the static string. The parameters can be easily adjusted to make the confining scale be far from the singularity, thus rendering our analysis reliable. However, we note a caveat that there is another branch for the solution of the static string dynamics, wherein the Wilson loop does not show the confining behavior and the long string is drooped all the way down to the singularity. It is not clear how to interpret this branch or "phase" in terms of the renormalization group flow of the dual gauge theory, since the regular branch alone covers all the length scale of the dual gauge theory.

Finally it is natural to ask if there is any brane interpretation for our geometry. On this score, it may be tempting to identify the singularity of the global deformation as a collection of unstable D0 branes smeared over $S^{5}$. The reasoning follows from the fact that the singularity is pointlike and does not carry any Neveu-Schwarz (NS) or Ramond-Ramond (RR) charge, but sources the dilaton and gravity. A speculation on this point will be elaborated and expanded in the discussion.

The organization of our paper is as follows. In section 2, we set up the ansatz for our dilatonic deformations, and propose its interpretation in terms of the AdS/CFT correspondence. In section 3, we discuss the case of deformation in the global coordinates. Particular emphasis is put on the causality bound and the condition for the repulsion. For the most 
part, we solve the problem numerically and find the causality (lower) bound on the mass for any given $k$, whereas the condition for the repulsion is found exactly. Sections 4, 5 and 6 deal with the case of deformation in the Poincaré patch. We find the exact analytic solution and the simple causality bound. The condition for the repulsion turns out to be the same as that in the global coordinates. We show evidence for the confinement by calculating the Wilson loop (static string), and clarify its relation to the repulsion. We also compute the tension of the QCD string exactly. Final section is devoted to discussions, and an appendix is provided for the case of deformation in the hyperbolic slicing.

\section{Preliminary}

We consider the following simple non-supersymmetric dilatonic deformations of $\mathrm{AdS}_{5} \times S^{5}$ :

$$
\begin{aligned}
d s^{2} & =l^{2}\left[-h(r) g(r) d t^{2}+\frac{h(r)}{g(r)} d r^{2}+r^{2} d s_{3, K}^{2}+d \Omega_{5}^{2}\right] \\
\phi & =\phi(r) \\
F_{5} & =Q l^{5}\left(d \Omega_{5}+* d \Omega_{5}\right)
\end{aligned}
$$

where $d \Omega_{5}$ denotes the volume form of the five sphere and $l$ is the radius of undeformed $\mathrm{AdS}_{5}$ and $S^{5}$. The subscript $K$ in $d s_{3, K}^{2}$ labels the curvature of the maximally symmetric 3-dimensional space, and $Q$ is a constant. In our convention, the curvature $K$ is equal to +6 for sphere and -6 for hyperboloid. The isometry of this geometry in general case is an

$S O(2) \times G_{3} \times S O(6)$ subgroup of $S O(2,4) \times S O(6)$, where $G_{3}$ is the isometry group of the maximally symmetric 3 -dimensional space with curvature $K$, i.e. $S O(4)$ for $K>0, I S O(3)$ for $K=0$, and $S O(1,3)$ for $K<0$. The $S O(2)$ is the time translation symmetry whereas the $S O(6)$ is the isometry of $S^{5}$.

The equations of motion for the type IIB string are given by

$$
\begin{aligned}
\nabla^{2} \phi & =0 \\
d * F_{5} & =0 \\
R_{M N} & =\frac{1}{2} \partial_{M} \phi \partial_{N} \phi+\frac{g_{s}^{2}}{96} F_{M P Q R S} F_{N}{ }^{P Q R S},
\end{aligned}
$$

together with the Bianchi identity $d F_{5}=0$. The above ansatz leads to

$$
\begin{aligned}
\phi^{\prime} & =\frac{k / l^{3}}{r \psi}, \\
(\ln h)^{\prime} & =\frac{\left(k / l^{3}\right)^{2}}{6 r \psi^{2}}, \\
\psi^{\prime} & =r\left(\left(g_{s} Q l / 2\right)^{2} r^{2}+K / 3\right) h,
\end{aligned}
$$


where we have introduced $\psi=r^{2} g$ and $k$ is a constant. Thus the Einstein equations boil down to ${ }^{1}$

$$
r\left(\ln \psi^{\prime}\right)^{\prime}=\frac{6 r^{2}+\frac{K}{6}}{2 r^{2}+\frac{K}{6}}+\frac{\left(k / l^{3}\right)^{2}}{6 \psi^{2}} .
$$

Here the constant $Q$ for the 5 -form flux has been chosen to be $4 /\left(g_{s} l\right)$ so that the value of the flux, in our normalization, is $\left(2 \pi \sqrt{\alpha^{\prime}}\right)^{4} N$ with $l^{4}=4 \pi g_{s} N \alpha^{2}$.

In general the deformation $\delta \varphi$ of supergravity fields behaves asymptotically as

$$
\delta \varphi=a_{\Delta} r^{\Delta-4}+b_{\Delta} r^{-\Delta}+O\left(r^{-\Delta-1}\right)
$$

where the non-normalizable mode $a_{\Delta}$ corresponds, in the dual CFT, to the source [2, 3], whereas the normalizable mode $b_{\Delta}$ to the expectation value of the operator $\mathcal{O}_{\Delta}$ of dimension $\Delta$ [12, 13]. Here we are only interested in dimension four operators, since we are turning on only the dilaton which back reacts to the metric and thus we are deforming only massless modes. The dilaton corresponds to the supersymmetric completion of $\operatorname{Tr} F^{2}$, i.e. the $\mathcal{N}=4$ SYM Lagrangian density $\mathcal{L}_{C F T}$ itself. In our case, the dilaton behaves asymptotically as

$$
\phi=\phi_{\infty}-\frac{k / l^{3}}{4} r^{-4}+O\left(r^{-5}\right)
$$

where $\phi_{\infty}$ is not related to $k$ at all, as might be expected for the singular geometry. This translates, in the dual gauge theory, to the shift of the coupling constant

$$
\mathcal{L}_{\text {deformed }}=\left(1-\phi_{\infty}\right) \mathcal{L}_{C F T}
$$

and to the selection of a particular state with respect to which the operator $\mathcal{L}_{C F T}$ acquires the expectation value

$$
\left\langle\mathcal{L}_{C F T}\right\rangle=\frac{k / l^{3}}{4}
$$

The non-vanishing $k$ in general breaks all the supersymmetries. Furthermore, as we will see later, our deformation of metric in general leads to the non-vanishing expectation value for the energy

$$
\left\langle T_{00}\right\rangle \neq 0
$$

where its value is given by yet another parameter $A$ (or $\mu$ ) in our solutions.

Since the non-normalizable mode $\phi_{\infty}$ of the dilaton corresponds to a trivial shift of the coupling constant, the Lagrangian is essentially unaffected by the deformation. Hence $\phi_{\infty}$ does not play any relevant role. ${ }^{2}$ We only turn on the expectation value for the corresponding operators, which may be interpreted as a choice of the background, or the state, of the

\footnotetext{
${ }^{1}$ The $K=+6$ case was previously discussed in 20 .

${ }^{2}$ Thus we may choose a convenient value of $\phi_{\infty}$. In addition we shall set $l=1$ for the notational simplicity.
} 
field theory. The (non-supersymmetric) state thus chosen is basically characterized by two parameters $k$ and $A$ (or $\mu$ ).

Note also that the bosonic part of $\left\langle\mathcal{L}_{C F T}\right\rangle$ is given by

$$
\left\langle\mathcal{L}_{C F T}\right\rangle=\frac{1}{2 g_{Y M}^{2}}\left\langle\operatorname{Tr}\left(E^{2}-B^{2}\right)\right\rangle=\frac{k / l^{3}}{4} .
$$

Thus one may call the $k>0(k<0)$ case electric (magnetic) where the electric (magnetic) contribution wins over the magnetic (electric) one.

In the following, we shall discuss the case of $K=+6$ (global coordinates) and $K=0$ (Poincaré patch) in detail. We relegate the $K=-6$ case (hyperbolic slicing) to the Appendix.

\section{Global Deformation}

In this section we analyze the dilatonic deformation in the global coordinates of AdS. This is the case of $K=+6$ in Section 2. The dual boundary field theory lives on $R \times S^{3}$. The dilatonic deformation (2.1)-(2.3) corresponds to turning on constant expectation values of the Lagrangian density $\mathcal{L}_{C F T}$ as well as, as we will see below, of the energy $T_{00}$. We would like to emphasize that the state with such expectation values surely exists within $\mathcal{N}=4 \mathrm{SYM}$. Our ansatz (2.1)-(2.3) is the most general one that respects the properties of such state in the field theory. However, as we will show below, our proposed dual gravity solution inevitably contains a timelike naked singularity. Instead of giving up the singular solution, we will utilize the AdS/CFT correspondence as a diagnostic tool to determine which of singular solutions may be acceptable.

The equations of motion (2.5)-(2.7) cannot be solved analytically in this case. However, the asymptotic expansion of the solution around $r=\infty$ (boundary) and $r=0$ (singularity) can be found. The expansion around $r=\infty$ reads

$$
\begin{aligned}
& \psi=h_{\infty}\left(r^{4}+r^{2}-A\right)+\frac{k^{2}}{h_{\infty}}\left(\frac{1}{48 r^{4}}-\frac{11}{720 r^{6}}\right)+O\left(\frac{1}{r^{8}}\right), \\
& \phi=\phi_{\infty}-\frac{k}{h_{\infty}}\left(\frac{1}{4 r^{4}}-\frac{1}{6 r^{6}}+\frac{A+1}{8 r^{8}}\right)+O\left(\frac{1}{r^{10}}\right), \\
& h=h_{\infty}-\frac{k^{2}}{h_{\infty}}\left(\frac{1}{48 r^{8}}-\frac{1}{30 r^{10}}\right)+O\left(\frac{1}{r^{12}}\right) .
\end{aligned}
$$

It is easy to see that the overall factor $h_{\infty}$ in (3.1) can be absorbed by the rescaling of time in the metric (2.1). Hence one can set, without loss of generality, $h_{\infty}=1$, and the solution

depends on the three integration constants $k, \phi_{\infty}$ and $A$. The asymptotic expansion provides the initial conditions for the numerical study of the equations of motion. 
The behavior of the solution near $r=0$ is given by the following expansion:

$$
\begin{aligned}
& \psi=\psi_{0}\left(1+\frac{2 h_{0}}{2 \psi_{0}+\frac{k^{2}}{6 \psi_{0}}} r^{2+\frac{k^{2}}{6 \psi_{0}^{2}}}\right)+O\left(r^{4+\frac{k^{2}}{6 \psi_{0}^{2}}}, r^{2+\frac{k^{2}}{3 \psi_{0}^{2}}}\right), \\
& h=h_{0}\left(r^{\frac{k^{2}}{6 \psi_{0}^{2}}}-\frac{24 h_{0} k^{2}}{\psi_{0}^{3}\left(12+\frac{k^{2}}{\psi_{0}^{2}}\right)^{2}} r^{2+\frac{k^{2}}{3 \psi_{0}^{2}}}\right)+O\left(r^{4+\frac{k^{2}}{3 \psi_{0}^{2}}}\right), \\
& \phi=\phi_{0}+\frac{k}{\psi_{0}} \ln r+O\left(r^{2+\frac{k^{2}}{6 \psi_{0}^{2}}}\right) .
\end{aligned}
$$

For the solution with $k \neq 0$, one finds that there is always a naked (curvature) singularity at $r=0$. In addition the dilaton diverges; for $k>0(k<0)$, the string coupling goes to zero (infinity) at $r=0$. Note that one can set $h_{\infty}=1$ by the rescaling of time, but it is in general not possible to set both of $h_{\infty}$ and $h_{0}$ to one. One can prove, given $h_{\infty}>0$, the function $\psi(r)$ increases monotonically with $\psi_{0}>0$ for any nonvanishing $k$.

We will solve the equations of motion numerically with the initial conditions, given by $\phi_{\infty}, k$ and $A$, at some large cutoff $r_{f}$.

\subsection{The causality bound}

We have seen that there is a three parameter family of solutions. They all have the timelike naked singularity, but are the only possible candidates of the dual of the above-mentioned state in $\mathcal{N}=4 \mathrm{SYM}$. However, in order for them to be sensible geometries in the AdS/CFT correspondence, they have to satisfy the following causality condition: No information, sent from a point $\mathrm{P}$ to $\mathrm{Q}$ on the boundary, can propagate faster through the bulk than along the boundary. This is a causal consistency of holography in the bulk/boundary correspondence. In our particular case, the causality bound is found to be [1]

$$
\pi \leq 2 \int_{0}^{\infty} d r \frac{r^{2}}{\psi(r)}
$$

A typical singular spacetime that violates the causality bound (3.7) is the negative mass AdS-Schwarzschild black hole, as is easily seen from,

$$
\pi=2 \int_{0}^{\infty} \frac{d r}{r^{2}+1}>2 \int_{0}^{\infty} \frac{d r}{r^{2}+1-A / r^{2}}
$$

when $A$ is negative. Thus it is excluded from the AdS/CFT correspondence, as it should be.

In our application too, the negative $A$ is excluded. We can first show that $\psi(r ; A)>$ $\bar{\psi}(r ; A) \equiv r^{4}+r^{2}-A$ as follows: Let $\psi^{\prime}=\bar{\psi}^{\prime} \chi$. Then the equation (2.8) becomes

$$
r(\ln \chi)^{\prime}=\frac{k^{2}}{6 \psi^{2}}
$$


which is solved as

$$
\chi=e^{-\int_{r}^{\infty} d r \frac{k^{2}}{6 r \psi^{2}}} .
$$

Thus we obtain

$$
\psi=\bar{\psi}+\int_{r}^{\infty} d r \bar{\psi}^{\prime}\left(1-e^{-\int_{r}^{\infty} d r^{\prime} \frac{k^{2}}{6 r^{\prime} \psi^{2}}}\right) .
$$

This proves that $\psi(r ; A)>\bar{\psi}(r ; A)$. For $A<0$, this reads

$$
\pi>2 \int_{0}^{\infty} \frac{d r}{r^{2}+1+|A| / r^{2}}>2 \int_{0}^{\infty} d r \frac{r^{2}}{\psi(r ; A)} .
$$

Hence the causality condition is violated for $A<0$.

However, we find by a numerical study that the causality bound can actually be obeyed for a wide range of parameters $(A, k)$ with $A \geq 0$. The saturation of the bound provides a boundary curve $A(k)$ in the $(A, k)$ space, as shown in Figure 1. The causality condition is satisfied when $A \geq A(k) \geq 0$ with $A(k)$ being even in $k$.

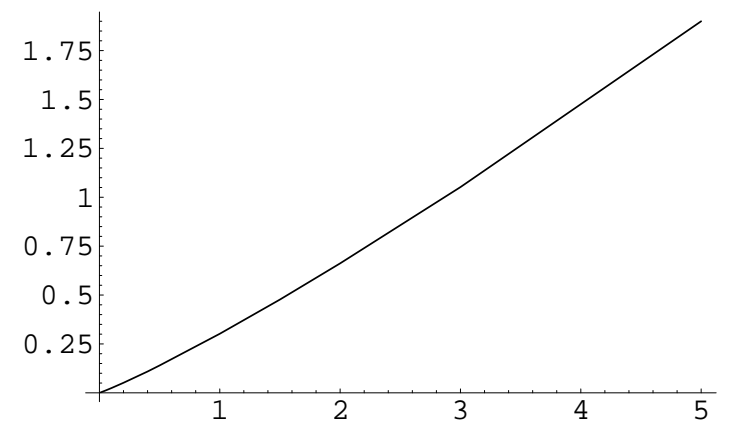

Figure 1: Critical ADM mass parameter $A(|k|)$ as a function of $|k|$.

The causality bound is, strictly speaking, a necessary condition for our spacetime to make sense in the AdS/CFT correspondence. But once the causality bound is cleared, we do not see any apparent obstruction for identifying our singular spacetime with the gravity dual of the above-mentioned state in the field theory, and we claim that our singular spacetime with $A \geq A(k)$ is indeed acceptable in the AdS/CFT correspondence.

\subsection{The repulson}

We have argued that our solution, albeit doomed to have the naked singularity, is still a viable gravity dual of a well-defined state in the field theory, as long as the causality condition $A \geq A(k)$ is met. Given this status, we now proceed to discuss physical properties of our singular geometry. The massive particle is a standard probe to study the characteristics of 
the geometry. In particular we consider the spherically symmetric geodesics of the massive particle, and will work in the string metric. The geodesic equations,

$$
\frac{d^{2} x^{\alpha}}{d \tau^{2}}+\Gamma_{\beta \gamma}^{\alpha} \frac{d x^{\beta}}{d \tau} \frac{d x^{\gamma}}{d \tau}=0
$$

reduce to the following first order equations,

$$
\frac{d t}{d \tau}=\frac{C r^{2}}{h \psi e^{\frac{\phi}{2}}},
$$

with $C$ being a positive integration constant, and

$$
\left(\frac{d r}{d \tau}\right)^{2}+V(r)=0
$$

where

$$
V(r)=\frac{\psi}{r^{2} h e^{\frac{\phi}{2}}}\left(1-\frac{C^{2} r^{2}}{\psi h e^{\frac{\phi}{2}}}\right) .
$$

This is a system of a zero-energy particle moving in one dimension under the potential (3.15). At small $r$, the potential behaves like

$$
V(r)=\frac{\psi_{0}}{h_{0} r^{\frac{1}{6}\left(\frac{k}{\psi_{0}}+\frac{3}{2}\right)^{2}+\frac{13}{8}}}\left(1-\frac{C^{2} r^{\frac{19}{8}-\frac{1}{6}\left(\frac{k}{\psi_{0}}+\frac{3}{2}\right)^{2}}}{h_{0} \psi_{0}}\right),
$$

where $\psi_{0}$ and $h_{0}$ were defined in (3.4) and (3.5).

The condition for the hard-core repulsion near $r=0$ is then given by

$$
-\frac{\sqrt{57}+3}{2}(\simeq-5.27) \leq \frac{k}{\psi_{0}} \leq \frac{\sqrt{57}-3}{2}(\simeq 2.27)
$$

Outside this range the naked singularity is attractive. Hence the quantity $k / \psi_{0}$ is a measure of whether the singularity is attractive or repulsive. In Figure 2, we plot $k / \psi_{0}$ for the critical values of $A(k)$ and $k$. It is easy to see that $k / \psi_{0}$ monotonically decreases, as $k$ decreases, for $k<0$, whereas there is no value of $(A(k), k)$ which satisfies the bound (3.17), for $k>0$. Hence, only for $0>k>-0.02$, the singularity can be repulsive, while saturating the causality bound.

\subsection{The ADM mass}

The parameter $A$ in our solution can be interpreted as the mass of the geometry. Indeed, using the counter term methods, one can compute the energy momentum tensor defined by 21, 22]

$$
T_{a b} \equiv K_{a b}-\gamma_{a b} K-3 \gamma_{a b}-\frac{1}{2}\left(R_{a b}(\gamma)-\frac{1}{2} \gamma_{a b} R(\gamma)\right)
$$




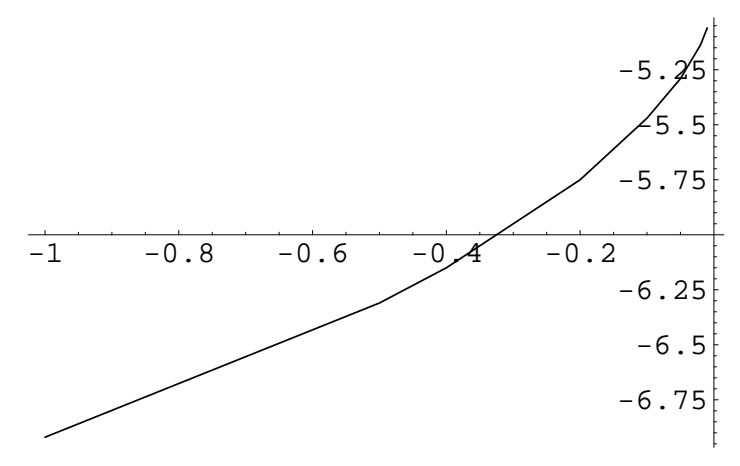

Figure 2: $k / \psi_{0}$ as a function of $k$ for the critical $A(k)$.

where $\gamma_{a b}$ is the induced metric on the boundary and $K_{a b}$ is the extrinsic curvature of the boundary. In our case, the mass is evaluated as

$$
M=\frac{3 \pi A}{8 G_{5}}+\frac{3 \pi}{32 G_{5}}
$$

where the second term represents the contribution of the Casimir energy on the sphere. Thus the mass for the excitation of our interest is given by

$$
M_{e x}=\frac{3 \pi A}{8 G_{5}} .
$$

Restoring $l$ dependence, one has

$$
\frac{l^{3}}{G_{5}}=\frac{2 N^{2}}{\pi}
$$

where $N$ denotes the number of D3 branes. For instance, the Casimir energy [21] is given by

$$
E_{c}=\frac{3 \pi l^{2}}{32 G_{5}}=\frac{3 N^{2}}{16 l}
$$

\subsection{Repulson vs confinement}

We would now like to argue that the infinitely strong repulsion discussed above might signal the confinement in the dual field theory. If one uses the static string probe corresponding to the Wilson loop, the hard-core repulsion near the singularity will keep the string away from the singularity for any given boundary separation of the quark-antiquark pair (see Figure 3). This may be expected on the basis of energetics, - this configuration minimizes the energy of the string.

Let us introduce the scale $r_{c}$ around which one sees an appreciable deviation of the geometry from the pure AdS case. We find it convenient to define the scale $r_{c}(<\infty)$ by 


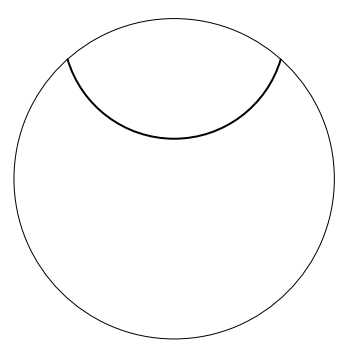

a) typical pure AdS string

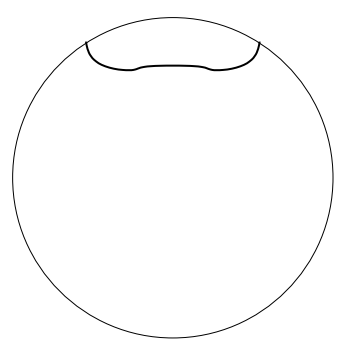

b) nonvanishing $k$ and large $r_{c}$

Figure 3: String configurations in the global deformation.

$\psi\left(r_{c}\right) / \psi_{A d S}\left(r_{c}\right)=1$ with $\psi_{A d S}(r)=r^{4}+r^{2}{ }^{3}$. This defines the scale at which the deviation is of order one. The scale $r_{c}$ is plotted in Figure 1 as a function of $|k|$ for the critical mass parameter $A(|k|)$. When the potential is repulsive, $r_{c}$ would be roughly the scale at which

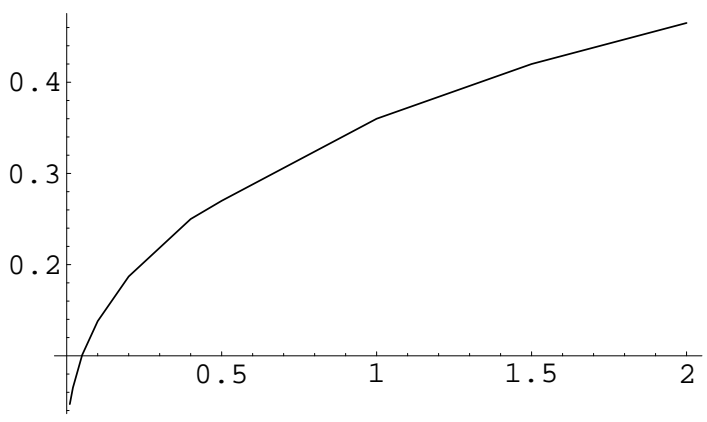

Figure 4: $r_{c}$ as a function of $|k|$ for $A(|k|)$.

the string turns around. As will be demonstrated below explicitly in the case of the Poincaré patch, when the separation of the quark-antiquark pair becomes larger and larger, more and more portion of the string stays around this scale. Hence, when the confinement occurs, it would happen below the energy scale,

$$
E_{\text {conf }} \sim r_{c}
$$

via the UV/IR relation of the AdS/CFT correspondence. ${ }^{4}$ In other words, when the quark-

\footnotetext{
${ }^{3}$ The function $\psi(r)$ approaches asymptotically to $r^{4}+r^{2}-A(|k|)$. Thus, for large but finite $r, \psi(r) / \psi_{A d S}(r)$ is less than one. Both $\psi(r)$ and $\psi_{A d S}(r)$ decrease as $r$ decreases, but $\psi(r)$ decreases slower than $\psi_{\text {AdS }}(r)$. Thus the point where the ratio becomes one is characteristic of the deformation.

${ }^{4}$ The UV/IR relation does also deviate from the pure AdS case. Thus the estimate of the confining scale is rough but within the accuracy of order one. As long as $r_{c}$ is much larger than the AdS radius, the estimate is reasonable.
} 
antiquark separation becomes larger than $1 / r_{c}$, the Wilson loop would show the area law. The Faraday flux between quark and antiquark at the boundary looks string-like with the size of its cross section being $1 / r_{c}$. All of these are a consequence of the repulsive force of our geometry. We illustrate the shape of string in Figure 3 as a cartoon, in the case when the potential is repulsive and $r_{c}$ is much larger than the AdS radius $l$.

However, our previous numerical result shows that for the repulsion and causality we have a rather narrow range, $0>k>-0.02$, which reads $r_{c}<0.065$. It then implies that, to see the confining behavior, the boundary separation must be much larger than $(1 / 0.065) l$, that is larger than the size of the AdS space and thus is not possible. Hence, in the global deformation, the confinement may not be seen clearly.

The study of the static string in Section 6 shows that the repulsion condition is actually relaxed to $k / \psi_{0}<-6$, as compared to the massive particle case. Since the condition is by nature local in the bulk geometry, one might expect that the same may be true for the global deformation. However, even with this relaxed condition, $r_{c}$ is still much smaller than one. Thus the conclusion concerning the confinement seems still negative. The conclusive answer would require a detailed numerical study of the behavior of the string connecting quark and antiquark in the global coordinates.

\section{Deformation in the Poincaré Patch}

In this section we consider a deformation of $\mathrm{AdS}_{5} \times S^{5}$ in the Poincaré patch, thus keeping the symmetry of $S O(2) \times I S O(3) \times S O(6) \subset S O(2,4) \times S O(6)$. The $S O(2)$ is again the time translation symmetry, and the $I S O(3)$ is the Poincaré symmetry of the Euclidean 3dimensional plane. This case corresponds to $K=0$ in our ansatz (2.1)-(2.3). It turns out that we can analytically obtain the exact solution in this case. In particular the exact analytic soluion allows us to show evidence for the confinement in the dual gauge theory, as we will see in Section 6. The dual $\mathcal{N}=4 \mathrm{SYM}$ theory lives on $3+1$ dimensional flat space in this case. As discussed in Section 2, the field theory background is nontrivial, on which the Lagrangian density $\mathcal{L}_{C F T}$ and the energy $T_{00}$ acquire constant expectation values. The backgrounds parameterized by different values of $k$ and $\mu$ are separated by the super-selection, and it costs an infinite amount of energy to interpolate them.

Our main equation (2.8) takes a particularly simple form,

$$
r\left(\ln \psi^{\prime}\right)^{\prime}=3+\frac{k^{2}}{6 \psi^{2}},
$$

where we set the radius $l$ to unity. The AdS boundary is located at $r=\infty$ and we require the asymptotically AdS condition as

$$
r^{-4} \psi \quad \rightarrow \quad 1
$$


as $r \rightarrow \infty$. The function $\psi^{\prime}$ is positive definite and its magnitude decreases monotonically as $r$ decreases. Moreover one can show that $\psi$ cannot become zero anywhere. Thus $\psi$ is convex, monotonically decreasing and remains positive definite, as $r$ decreases.

Indeed one can find the exact solution, which is given implicitly by

$$
r=(y-b)^{\frac{1-a}{8}}(y+b)^{\frac{1+a}{8}},
$$

where

$$
a \equiv\left(1+\frac{k^{2}}{6 \mu^{2}}\right)^{-\frac{1}{2}}, \quad b \equiv \frac{\mu}{2}\left(1+\frac{k^{2}}{6 \mu^{2}}\right)^{\frac{1}{2}} .
$$

with $y=\psi+a b$. Using (2.5)-(2.7), we get

$$
h=\frac{1}{y-a b}(y-b)^{\frac{1+a}{2}}(y+b)^{\frac{1-a}{2}},
$$

and

$$
\phi=\phi_{\infty}+\frac{k}{8 b} \ln \left(\frac{y-b}{y+b}\right) .
$$

In the limit $\mu \rightarrow 0$, we have $a \rightarrow 0$ and $b \rightarrow \pm|k| /(2 \sqrt{6})$, reducing to the solution found in [8, 9, 10].

\subsection{The causality bound}

The causality bound in this case is considerably simpler than that in the global deformation. Again we compare two paths connecting two boundary points $\mathrm{P}$ and Q, one along the boundary and the other through the bulk. The bulk path does not have to be geodesic. Then the holographic causality condition requires simply that $h g / r^{2} \leq 1$. Since

$$
h g / r^{2}=\left(\frac{y-b}{y+b}\right)^{a}
$$

the causality restricts $b \geq 0$, which in turn implies $\mu \geq 0$. Later we shall show that this corresponds to the requirement of the positive semi-definite mass density. Hence from now on we consider only the case of positive $\mu$.

\subsection{The repulson}

As in the case of the global deformation, we probe the singularity by the massive particle. We will again find the condition for the repulsion which turns out to be the same as that of the global deformation. The spherically symmetric geodesics amount again to a particle motion in the potential

$$
V(r)=\frac{g}{h e^{\frac{\phi}{2}}}\left(1-\frac{C^{2}}{g h e^{\frac{\phi}{2}}}\right)
$$


Note that

$$
U_{1} \equiv g h e^{\frac{\phi}{2}}=e^{\frac{1}{2} \phi_{\infty}} \frac{(y-b)^{\frac{k}{16 b}+\frac{3 a+1}{4}}}{(y+b)^{\frac{k}{16 b}+\frac{3 a-1}{4}}}
$$

and

$$
U_{2} \equiv \frac{h e^{\frac{\phi}{2}}}{g}=\frac{e^{\frac{1}{2} \phi_{\infty}}}{(y-a b)^{2}} \frac{(y-b)^{\frac{k}{16 b}+\frac{3+a}{4}}}{(y+b)^{\frac{k}{16 b}+\frac{3-a}{4}}} .
$$

We are interested in the behavior of the potential as $r \rightarrow 0$. This corresponds to the limit $y \rightarrow b$. Since $\frac{k}{16 b}+\frac{3+a}{4}>0$ always holds, $U_{2} \rightarrow 0^{+}$as $r \rightarrow 0$. Furthermore, if

$$
\frac{k}{16 b}+\frac{3 a+1}{4}<0
$$

then $U_{1} \rightarrow \infty$, and the potential will have the hard-core repulsion near the singularity at $r=0$. Otherwise, $U_{1} \rightarrow 0^{+}$, and we will have the infinitely attractive singularity at $r=0$.

The condition (4.11) is solved as

$$
k<-2(9+\sqrt{57}) \mu
$$

Let us now work out the asymptotic behavior of the solution. As $r \rightarrow 0$,

$$
\psi_{0} \equiv \psi(0)=\frac{|\mu|}{2}\left(\sqrt{1+\frac{k^{2}}{6 \mu^{2}}}-1\right) .
$$

Using this relation and (4.12), one can show that the repulsion condition is expressed as

$$
-\frac{1}{2}(3+\sqrt{57})(\simeq-5.27)<\frac{k}{\psi_{0}} \quad(\text { with } k<0)
$$

which is in agreement with (3.17) of the global deformation. In addition, it is straightforward to show that

$$
h \rightarrow 1+O\left(r^{-8}\right), \quad g / r^{2} \rightarrow 1-\mu r^{-4}+O\left(r^{-8}\right), \quad \phi \rightarrow \phi_{\infty}-\frac{k}{4} r^{-4}+O\left(r^{-8}\right),
$$

as $r \rightarrow \infty$. Incidentally, from the correspondence of (2.12), the negative $k$ corresponds to the magnetic background, as noted in the end of Section 2 .

\subsection{The ADM mass}

The parameter $\mu$ can be interpreted as the mass parameter of the geometry like $A$ in the global deformation. Using (3.18), we identify the mass of the system as

$$
M=\frac{3 \mu}{16 \pi G} \int d \vec{x}
$$


Therefore the mass density of the boundary CFT is given by

$$
T_{00}^{b d}=\frac{3 \mu}{16 \pi G}=\frac{3 N^{2} \mu}{8 \pi^{2} l^{4}}
$$

where we have restored $l$ dependence with the dimensionless quantity $\mu$ in the unit of $l$.

In conclusion, for the Poincaré patch solution the causality implies just the exclusion of the negative mass density. For the causal case, we expect to have in general two phases depending on the expectation value of $\mathcal{L}_{C F T}$. When $k<-2(9+\sqrt{57}) \mu$, the theory is confining, as we will show in Section 6 .

\section{Nature of Singularity}

In this section we discuss the nature of singularity in the Poincaré deformation. In the global deformation, we have found that the singularity is timelike. In the present case, one finds that $h g=0$ and $h / g=0$ at the singularity $y=b$. Thus at first glance the singularity might appear lightlike. However, as pointed out in [23], this conclusion could be premature and merely an artifact of the particular coordinate system. We now show that this is indeed the case. Note that the metric in fact can be represented in terms of $y$ coordinate by

$$
d s^{2}=(y-b)^{\frac{1-a}{4}}(y+b)^{\frac{1+a}{4}}\left(-\left(\frac{y-b}{y+b}\right)^{a} d t^{2}+\frac{d y^{2}}{16(y-b)^{\frac{5-a}{4}}(y+b)^{\frac{5+a}{4}}}+d \vec{x}^{2}\right)+d \Omega_{5}^{2} .
$$

To see what is happening in the Penrose diagram in $(t, y)$-plane, let us introduce the coordinate $w$ defined by

$$
w(y)=\int_{y}^{\infty} \frac{d \tilde{y}}{(\tilde{y}-b)^{\frac{5+3 a}{8}}(\tilde{y}+b)^{\frac{5-3 a}{8}}} .
$$

The range of $w$ is given by $\left[0, w_{0}\right]$ with $w_{0}=w(b)>0$ for $k \neq 0$. In terms of this variable, the metric with fixed $\vec{x}$ and the angular coordinates becomes

$$
d s^{2}=(y-b)^{\frac{1+3 a}{4}}(y+b)^{\frac{1-3 a}{4}} d(w-t) d(w+t) .
$$

Introducing further $u_{ \pm}=\tan ^{-1}(w \pm t)$, the metric becomes

$$
d s^{2}=(y-b)^{\frac{1+3 a}{4}}(y+b)^{\frac{1-3 a}{4}} \sec ^{2} u_{+} \sec ^{2} u_{-} d u_{+} d u_{-} .
$$

The range of $u_{ \pm}$is given by $\left[-\frac{\pi}{2}, \frac{\pi}{2}\right]$ with $0 \leq u_{+}+u_{-}$and $\tan u_{+}+\tan u_{-} \leq 2 w_{0}$. The Penrose diagram is depicted in Figure 5. Hence it is now clear that the trajectory of singularity is timelike.

Also for any finite size box in the direction of $\vec{x}$, the singularity is essentially pointlike due to the scale factor $r^{2}$ that becomes zero as one approaches the singularity. 


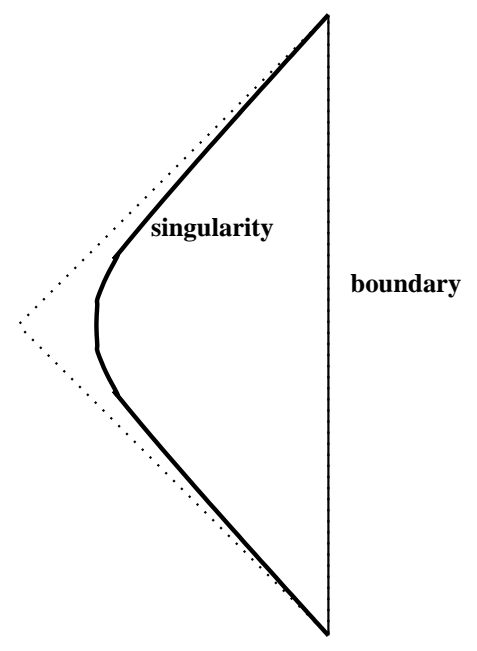

Figure 5: The Penrose diagram for the Poincaré deformation.

\section{Wilson Loop and Confinement}

We argued in Section 3.4 that the infinitely strong repulsion is indicative of the confinement in the dual gauge theory. Our argument was based on an extrapolation of the behavior of the static string from that of the massive particle in the global deformation. In this section, exploiting the exact analytic form of the solution in the Poincaré patch, we will make our claim precise, by directly calculating the static string corresponding to the Wilson loop.

The action for the fundamental string is given by

$$
S=-\frac{1}{2 \pi \alpha^{\prime}} \int d \tau d \sigma \sqrt{-\operatorname{det}\left(g_{\mu \nu} \partial_{a} X^{\mu} \partial_{b} X^{\nu}\right)},
$$

where $g_{\mu \nu}$ is the string frame metric. We assume that the Wilson loop is static and choose the gauge $\tau=t$ and $\sigma=y$. We shall consider the case where the Wilson loop is independent of $x_{2}$ and $x_{3}$. The Nambu-Goto Lagrangian becomes

$$
L=-\int d y \sqrt{A(y)\left(B(y)+C(y)(d x / d y)^{2}\right)},
$$

where we are considering the string frame metric of the form

$$
d s^{2}=-A(y) d t^{2}+B(y) d y^{2}+C(y) d x^{2},
$$

which is found from (5.1) and (4.6) as

$$
\begin{aligned}
& A(y)=e^{\phi_{\infty} / 2}(y-b)^{\frac{1+3 a}{4}+\frac{k}{16 b}}(y+b)^{\frac{1-3 a}{4}-\frac{k}{16 b}}, \\
& B(y)=\frac{1}{16} e^{\phi_{\infty} / 2}(y-b)^{-1+\frac{k}{16 b}}(y+b)^{-1-\frac{k}{16 b}}, \\
& C(y)=e^{\phi_{\infty} / 2}(y-b)^{\frac{1-a}{4}+\frac{k}{16 b}}(y+b)^{\frac{1+a}{4}-\frac{k}{16 b}} .
\end{aligned}
$$


The equation of motion is given by

$$
\frac{d}{d y}\left(\frac{\sqrt{A} C d x / d y}{\sqrt{B+C(d x / d y)^{2}}}\right)=0 .
$$

Integrating this once, we get

$$
\frac{\sqrt{A} C d x / d y}{\sqrt{B+C(d x / d y)^{2}}}= \pm q^{-2}
$$

A further integration leads to the solution

$$
x-x_{0}= \pm \int d y \frac{\sqrt{B}}{\sqrt{C} \sqrt{q^{4} A C-1}} .
$$

To understand what it implies, let us rewrite $(6.6)$ in the form

$$
\left(\frac{d y}{d x}\right)^{2}+\mathcal{V}(y)=0
$$

with the potential

$$
\mathcal{V}(y)=\frac{C}{B}\left(1-q^{4} A C\right)=16 \frac{(y-b)^{\frac{5-a}{4}}}{(y+b)^{\frac{-5-a}{4}}}\left(1-e^{\phi_{\infty}} q^{4} \frac{(y-b)^{\frac{1+a}{2}+\frac{k}{8 b}}}{(y+b)^{\frac{-1+a}{2}+\frac{k}{8 b}}}\right) .
$$

This can be viewed as a zero-energy particle moving in one dimension under the potential $\mathcal{V}$, regarding the coordinate $x$ as the 'time'.

The confinement will occur when the zero-energy 'particle' spends an arbitrarily large 'time', when it approaches the turning point denoted by $y_{0}$. At the turning point, one has $d y / d x=0$. Thus $\mathcal{V}\left(y_{0}\right)=0$, which implies that

$$
q_{0}^{4} A\left(y_{0}\right) C\left(y_{0}\right)=1
$$

for an appropriate choice of the integration constant $q=q_{0}$. The condition of arbitrarily large 'time' spending may be fulfilled if $\mathcal{V}^{\prime}\left(y_{0}\right)=0$. This leads to

$$
\left.(\ln A C)^{\prime}\right|_{y=y_{0}}=\frac{y_{0}+a b+k / 4}{y_{0}^{2}-b^{2}}=0
$$

where the condition $\mathcal{V}\left(y_{0}\right)=0$ is used. For the existence of the solution in the range $y \in(b, \infty)$, one has to satisfy

$$
y_{0}-b=-a b-k / 4-b \equiv 2 b \beta>0 .
$$

because $y>b$. The solution is given by

$$
k<-12 \mu
$$


Then $\mathcal{V}\left(y_{0}\right)=0$ is satisfied by choosing the integration constant $q$ as

$$
q_{0}^{4}=\frac{1}{2 b} \beta^{\beta}(1+\beta)^{-(1+\beta)} e^{-\phi_{\infty}} .
$$

If these requirements are met, the potential may be approximated by

$$
\mathcal{V}=-\kappa^{2}\left(y-y_{0}\right)^{2}+\cdots
$$

near the turning point for some constant $\kappa$. The solution of $(6.7)$ is given in the form of $x-x_{0} \simeq \pm \frac{1}{\kappa} \ln \left|y-y_{0}\right|$, which is consistent with the condition of arbitrarily large time spent by the particle approaching $y_{0}$.

Let us now describe how the string behaves depending on various integration constants and parameters.

\subsection{Coulombic case}

For $\beta<0$ (or equivalently $k>-12 \mu$ ) and any $q$, the potential starts from large negative values for large $y$, crosses zero at $y=y_{0}$, turns around at some $y\left(<y_{0}\right)$ and approaches zero as $y \rightarrow b$, as depicted in Figure 6 .

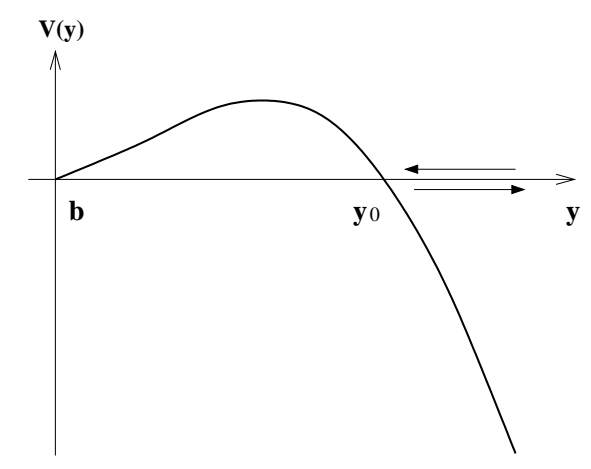

Figure 6: The shape of the potential for $\beta<0$.

The zero-energy particle turns around at $y=y_{0}$ without spending much 'time'. The behavior of the string is not much different from the pure AdS case where the quark-antiquark potential is Coulombic. Therefore this case corresponds to the Coulomb phase.

For $\beta>0$ and $q<q_{0}$, the shape of the potential is again similar to the pure AdS case. In particular for $q \ll q_{0}$, the shape of the string and potential is not much different from those 
in the pure AdS. The string remains in the asymptotic region where the geometry approaches the pure AdS spacetime (see Figure 0). For small $q$, the separation between the quark and antiquark is of the order of $q$ according to the IR/UV relation. The energy scale here is much higher than that of the confinement. Thus the quark-antiquark potential is essentially Coulombic.

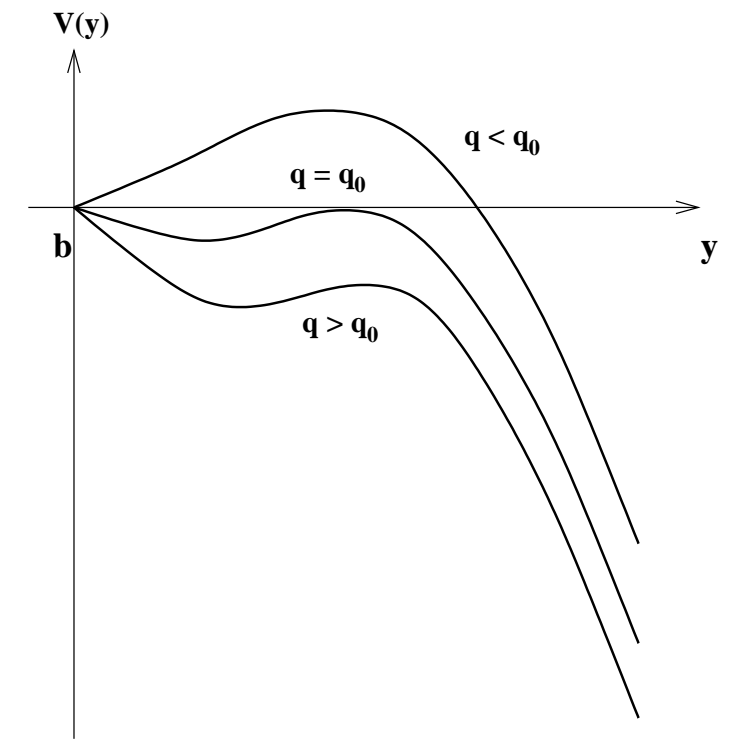

Figure 7: The shape of the potential for $\beta>0$.

\subsection{Confining case}

When $\beta>0$ and $q$ approaches $q_{0}$ from below, the string spends more and more 'time' near the turning point $y \sim y_{0}$. The separation of the quark and antiquark becomes larger and larger when one sends $q$ to $q_{0}$ from below because the 'time' spent near the turning point increases more and more, as depicted in Figure 8.

In the limit $q \rightarrow q_{0}$, we can compute the tension of the QCD string and the energy scale of the confinement. The energy of the string is given by

$$
E=\int d y \sqrt{A\left(B+C(d x / d y)^{2}\right)}=\int d x \sqrt{q^{4} A^{2} C^{2}}
$$




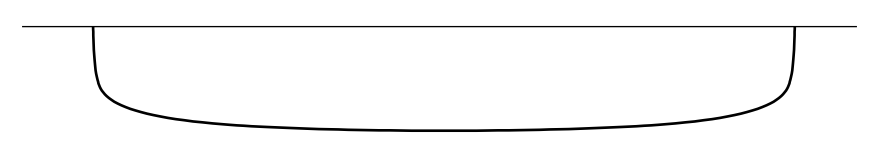

Figure 8: A string configuration representing confinement.

where we have used the equation of motion. The integral in fact diverges and one may regulate it by subtracting the self-energy of quark and antiquark, which is twice of the quark self-energy obtained by setting $x=0$ :

$$
E_{q}=\int_{b}^{\infty} d y \sqrt{A B}
$$

Then the quark-antiquark potential is given by

$$
V_{q \bar{q}}=2 \int_{y_{0}}^{\infty} d y \sqrt{A B} \sqrt{\frac{q^{4} A C}{q^{4} A C-1}}-2 \int_{b}^{\infty} d y \sqrt{A B} .
$$

Since $q_{0}^{4} A\left(y_{0}\right) C\left(y_{0}\right)=1$ and the string stays near the turning point for most of the 'time', we find from (6.16) the tension of the QCD string to be

$$
T_{Q C D}=\sqrt{A\left(y_{0}\right) C\left(y_{0}\right)}=q_{0}^{-2}=\sqrt{\mu} \frac{(1+\beta)^{\frac{1+\beta}{2}}}{\sqrt{a} \beta^{\frac{\beta}{2}}} e^{\phi_{\infty} / 2}
$$

with $\Delta E=T_{Q C D} \Delta x$. This sets the scale of the confinement, and was previously calculated in $[8]$ in the $\mu \rightarrow 0$ limit. When the separation $L$ is much larger than $q_{0}=1 / \sqrt{T_{Q C D}}$, we are in the confining phase.

When the integration constant $q$ is larger than $q_{0}$ with $\beta>0$, one finds that the turning point corresponds to $y=b$ and the string touches the singularity at the turning. However the string coupling becomes large near $y=b$ so that we cannot make a definite statement as to what is physically happening in this regime. Note also that the regular branch $q \leq q_{0}$ alone covers all the energy scale of the boundary field theory.

Finally if one rewrites the condition for the confinement of (6.13) in terms of $\frac{k}{\psi_{0}}$ using (4.13), one gets a weaker condition,

$$
-6<\frac{k}{\psi_{0}} \quad(\text { with } k<0)
$$

than the one in 4.14). Since this condition is by nature local in the bulk geometry, one may expect that the same may be true for the global deformation. 


\subsection{The Mass Gap}

As further evidence for the confinement, we will show there exists a mass gap in the dual gauge theory, following [24]. The same calculation was done in 8 for $\mu=0$. Here we will generalize their result to the case of non-vanishing $\mu(>0)$.

We consider the fluctuation $\delta \phi$ of the dilaton about our background, and see if $\delta \phi$ has the discrete spectrum with a mass gap. To show the mass gap, it is sufficient to think of the $\mathrm{s}$-wave on the five sphere, thus setting $\delta \phi=\varphi(z) e^{i \omega t}$. Then the fluctuation mode obeys

$$
\frac{d^{2}}{d z^{2}} \varphi+V(z) \varphi=0
$$

with

$$
V=\frac{\omega^{2}}{16} \frac{e^{\frac{3}{2} a b z}}{\sinh ^{\frac{3}{2}} b z} .
$$

The coordinate variable $z$ is related to $y$ by

$$
z=-\int \frac{d y}{y^{2}-b^{2}}=\frac{1}{2 b} \ln \left(\frac{y+b}{y-b}\right)
$$

The existence of the gap for $k / \mu=-\infty(\mu \rightarrow 0)$, was shown in [8], and a few lowest values of $\omega^{2}=m^{2} l^{2}$ for $a=0$ are given in the Table 1 of their paper.

We have carried out a numerical analysis which shows the mass gap for $\mu>0$ with some upper bound on $\mu$, as anticipated from the condition (6.13) for the confinement. To illustrate this we plot the mass of the lowest mass $(l=0)$ glueball as a function of $a$ in units where $b=1$. The parameters $a, b$ are related to $k, \mu$ by (4.4). Note that the glueballs cease to exist for $a=1$, indicating no confinement.

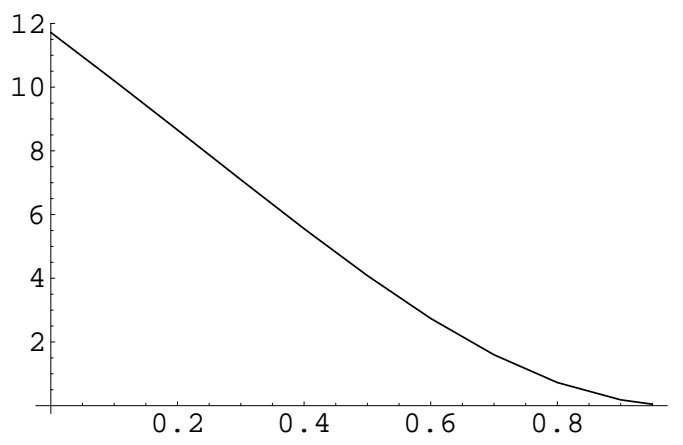

Figure 9: Mass of lowest mass glueball as function of $a$. 


\section{Discussions}

In this paper, we have proposed gravity duals of certain non-supersymmetric backgrounds or states in $\mathcal{N}=4 \mathrm{SYM}$ which give the non-vanishing expectation values to the dimension four operators, $\mathcal{L}_{C F T}$ and $T_{00}$. The backgrounds are homogeneous and time-independent, and respect the full $S O(6)$ R-symmetry. Our proposed gravity duals are of non-supersymmetric dilatonic deformation. In particular we have studied two cases in detail - the global and Poincaré deformations, whose dual field theory lives on $R \times S^{3}$ and $3+1$ dimensional Minkowski spacetime respectively.

We have carried out numerical studies, for the most part, in the case of the global deformation, whereas we have found the exact analytic solution for the Poincaré deformation. It is shown that the naked singularity is inevitable for this class of deformations and it is in fact timelike. Then the causality bound of 11] is used as a criterion to determine which of our singular geometries may make sense in string theory. This leads us to the positive lower bound $A(k)$ on mass $A$ for a given $k$ in the global deformation, whereas to the positivity of mass $\mu$ (irrespective of $k$ ) in the Poincaré deformation. The causality bound is predominantly determined by the contribution from the spacetime region far off the singularity. Thus our estimate of the causality bound should be plausible, although the supergravity approximation breaks down near the singularity. It is our claim that the singular geometries we have found are sensible in the AdS/CFT correspondence, as long as the causality bound is satisfied, irrespective of the validity of our approximation, while the accuracy of our analysis is only reliable away from the singularity.

Another criterion for a solution to be physical is given in Ref. [25], which states that large curvature naked singularities are allowed only if the scalar potential is bounded from above in the solution. If we accept this criterion, our solution is physical irrespective of whether $\mu=0$ or not since the scalar potential is zero identically.

Our proposed criterion for the sensibility of geometry with a naked singularity crucially relies on the existence of the well-defined dual field theory state. Also there is no credible way to argue the stability of our geometry within the supergravity approximation. The question of stability can thus be checked only through the confirmation of the existence of the field theory state and study of its properties.

Here we give a brief argument to bolster the existence of such a state. Let us first consider the case $\mu=0$. In this case the Lorentz invariance is restored, as can be seen, for example, from the metric (5.1) with $a=0$. This is consistent with the dual field theory description where $\left\langle T_{00}\right\rangle=0$ and $\left\langle T_{0 i}\right\rangle=\left\langle T_{i j}\right\rangle=0$. Also having the expectation value for the Lagrangian density, $\langle\mathcal{L}\rangle=k / 4$, respects the Lorentz invariance. Since $T_{\mu \nu}=F_{\mu \alpha} F_{\nu}{ }^{\alpha}-\frac{1}{4} \eta_{\mu \nu} F^{2}$ for $X^{I=1 \cdots 6}=0$, these imply that $-\left\langle F_{0 \alpha} F_{0}{ }^{\alpha}\right\rangle=\left\langle F_{i \alpha} F_{i}{ }^{\alpha}\right\rangle=\frac{1}{4}\langle\mathcal{L}\rangle=k / 16$ and $\left\langle F_{0 \alpha} F_{i}{ }^{\alpha}\right\rangle=$ 
$\left\langle F_{i \alpha} F_{j}^{\alpha}\right\rangle=0$ for $i \neq j$. Such a state can exist, though its explicit construction is beyond our scope. Raising the value of $\mu$ breaks the Lorentz invariance but keeps the $S O(3)$ rotation symmetry. In fact it yields $\left\langle T_{00}\right\rangle=\frac{3 N^{2} \mu}{8 \pi^{2}}$ and $\left\langle T_{0 i}\right\rangle=\left\langle T_{i j}\right\rangle=0$ in the dual field theory, matching the symmetry on both sides. By a similar argument, again we do not see any contradiction for the existence of such a state.

It seems also suggesting that our geometry is stable, since the dual field theory state is an energy eigenstate and thus cannot decay into anything else by the unitary evolution driven by the $\mathcal{N}=4$ SYM Hamiltonian.

As a physical implication, we expect that the confinement may occur in our non-supersymmetric dilatonic deformation, since the deformation introduces a mass scale in the theory. We have argued that, for the confinement to actually happen, it would be signaled by the hard-core repulsion from the naked singularity for one possibility. We have shown that there is a certain range of parameters for the global/Poincaré deformations in which the geometry indeed exhibits the strong repulsion. Both in the global and Poincaré deformations, we find an overlap between the ranges of parameters for the repulsion and causality.

However, it turns out that the would-be confining scale in the global case is too large compared to the size $l$ of the system. Therefore the confinement representing a linear potential between quark and antiquark as its defining property, cannot be seen for the global deformation. On the other hand, in the Poincaré deformation, it is shown that the confinement indeed occurs, for a certain range of parameters, by the explicit calculation of the static string corresponding to the Wilson loop. The confinement in this case is in fact a consequence of the strong repulsion from the singularity as depicted in Figure 8 .

What would be the objects located at the origin of AdS space in the global deformation? Clearly these objects are zero dimensional, which are distributed over $S^{5}$ uniformly. They produce no R-R flux and only accompany the non-vanishing dilaton. Furthermore these objects are non-supersymmetric. There are only two candidates in the IIB closed string theory with these specifications. One is the unstable D0-brane and the other is the AdSSchwarzschild black hole. Because our solution does not involve any horizon, we may exclude the possibility of the black hole. Thus it is tempting to identify the timelike naked singularity with unstable D0-branes, sitting at the center of AdS space.

The above solutions involve three free parameters, two of which are physically relevant. The parameter $k$, the strength of the dilaton, would be related with the number of D0-branes. The parameter $A$, the ADM mass, should represent the total eigen-energy of the collection of unstable D0-branes (see [26, 27] for related discussions).

It is interesting to try to understand the physics of the unstable D0-branes more deeply. The analysis of the rolling tachyon boundary CFT [28] for the decay of an unstable brane [29] 
suggests that the D0-branes decay into pressureless dust. The energy-momentum tensor $T_{00}=\frac{\tau_{0}}{2}(\cos 2 \pi \lambda+1)$ depends on a parameter $\lambda$ of the boundary CFT. A particular simple interpretation of the tachyon matter was suggested in [30], where it was argued that the unstable brane decays primarily into closed string modes which (at weak coupling) are long lived. Furthermore the massive string states that have nonrelativistic velocities are localized near the locus of the D0-brane for a long time. Note that for $k>0$ the string coupling becomes arbitrarily weak at the location of the singularity and one might expect that the tachyon matter does not diffuse at all. There is also a new feature in AdS, where massive states are confined by the AdS curvature from propagating away from the center of AdS. It would be interesting to make this stringy resolution of the singularity more precise. Unfortunately the exact boundary states are only known in flat space and not available for the $A d S_{5} \times S^{5}$ background. With present techniques, only an analysis using the tachyon effective action coupled to type IIB supergravity in the spirit of [31, 32] seems feasible. We will leave this interesting question for future work.

In particular we do not know the exact relation of the parameters $A$ and $k$ of the supergravity solution to the parameters of the D0-branes. It is suggestive to interpret the marginal case, $A(k)$, will correspond to the unstable D0-branes in their ground state.

The decay process of the tachyon could be described by an S-brane type of solutions 33, 34, 35, 36. In the past the formation of naked singularities in numerical relativity using finely tuned incoming dilaton and graviton excitations has been studied in detail [37]. A possible scenario is to consider an incoming pulse of dilaton and graviton which forms a naked singularity is actually an S-brane process of the formation of an unstable D0-brane. The solution should correspond to time-dependent space-like brane in AdS space. One possible line of further investigation is to study such processes numerically (see [38, 39] for first steps in this direction). It would also be interesting to find the corresponding solutions and study their relevance to the present problem.

In conclusion we have found a very simple example of the confinement of YM theories via AdS/CFT. The bulk mechanism behind the confinement is speculated due to the presence of the unstable D0-branes.

\section{Acknowledgement}

DB is supported in part by KOSEF ABRL R14-2003-012-01002-0 and KOSEF R01-2003-00010319-0. The work of MG was supported by NSF grant 0245096. Any opinions, findings and conclusions expressed in this material are those of the authors and do not necessarily reflect the views of the National Science Foundation. The work of SH was supported in part by the Israel Science Foundation under grant no. 101/01-1, and that of NO was supported in part 
by Grants-in-Aid for Scientific Research Nos. 12640270 and 02041.

\section{A. Hyperbolic Slicing}

In sections 3 and 4, we study in detail the dilatonic deformations Eqs.(2.1)-(2.3) in the global coordinates and the Poincaré patch respectively. In this appendix, we briefly discuss the case of $K=-6$, i.e. the hyperbolic slicing.

Our main equation (2.8) in this case is given by

$$
r\left(\ln \psi^{\prime}\right)^{\prime}=\frac{6 r^{2}-1}{2 r^{2}-1}+\frac{k^{2}}{6 \psi^{2}} .
$$

To see what is happening, let us look at the case where $k=0$. The solution reads

$$
g=r^{2}-1-A / r^{2}, \quad h=1 .
$$

The claim is that the singularity is a three-dimensional hypersurface which is extended to the boundary.

To show it, we choose the metric of 3-dimensional hyperboloid to be

$$
d s_{3, K=-6}^{2}=\frac{1}{\tilde{w}^{2}}\left(d \tilde{w}^{2}+d x_{1}^{2}+d x_{2}^{2}\right) .
$$

In the case where $A=0$, introducing new coordinates

$$
\tilde{t}=\tilde{w} \sin \theta \sinh t, \quad \tilde{x}_{3}=\tilde{w} \sin \theta \cosh t, \quad \tilde{z}=\tilde{w} \cos \theta,
$$

with $r=1 / \cos \theta$, one finds that the metric (2.1) is

$$
d s^{2}=\frac{1}{\tilde{z}^{2}}\left(-d \tilde{t}^{2}+d \tilde{z}^{2}+d x_{1}^{2}+d x_{2}^{2}+d \tilde{x}_{3}^{2}\right)+d \Omega_{5}^{2},
$$

i.e. the same as that in the Poincaré patch. When $A$ is non-vanishing, the event horizon appears at the fixed $\theta=\theta_{0}$, determined by

$$
K(\theta)=r^{2}-1-A / r^{2}=\tan ^{2} \theta-A \cos ^{2} \theta=0 .
$$

For $t=0$ with $\theta=\theta_{0}$, it describes a three dimensional surface extended along $x_{1}, x_{2}$ and $\left(\tilde{z}, \tilde{x}_{3}\right)=\tilde{w}\left(\cos \theta_{0}, \sin \theta_{0}\right)$. For $t \neq 0$, one finds that the shape is time dependent in the standard coordinates of the Poincaré patch in (1.5).

\section{References}

[1] J. M. Maldacena, "The large N limit of superconformal field theories and supergravity," Adv. Theor. Math. Phys. 2, 231 (1998) [Int. J. Theor. Phys. 38, 1113 (1999)] [arXiv:hep-th/9711200]. 
[2] S. S. Gubser, I. R. Klebanov and A. M. Polyakov, "Gauge theory correlators from non-critical string theory," Phys. Lett. B 428, 105 (1998) [arXiv:hep-th/9802109].

[3] E. Witten, "Anti-de Sitter space and holography," Adv. Theor. Math. Phys. 2, 253 (1998) [arXiv:hep-th/9802150].

[4] G. 't Hooft, "Dimensional Reduction In Quantum Gravity," arXiv:gr-qc/9310026.

[5] L. Susskind, "The World as a hologram," J. Math. Phys. 36, 6377 (1995) [arXiv:hep-th/9409089].

[6] D. Bak, M. Gutperle and S. Hirano, "A dilatonic deformation of $\operatorname{AdS}(5)$ and its field theory dual," JHEP 0305, 072 (2003) [arXiv:hep-th/0304129].

[7] D. Z. Freedman, C. Nunez, M. Schnabl and K. Skenderis, "Fake supergravity and domain wall stability," arXiv:hep-th/0312055.

[8] S. S. Gubser, "Dilaton-driven confinement," arXiv:hep-th/9902155.

[9] A. Kehagias and K. Sfetsos, "On running couplings in gauge theories from type-IIB supergravity," Phys. Lett. B 454, 270 (1999) [arXiv:hep-th/9902125].

[10] S. Nojiri and S. D. Odintsov, "Curvature dependence of running gauge coupling and confinement in AdS/CFT correspondence," Phys. Rev. D 61, 044014 (2000) [arXiv:hep-th/9905200].

[11] M. Kleban, J. McGreevy and S. Thomas, "Photon telegram to S. Claus: Consequences for holography and causality in AdS," JHEP 0403, 006 (2004) [arXiv:hep-th/0112229].

[12] V. Balasubramanian, P. Kraus and A. E. Lawrence, "Bulk vs. boundary dynamics in anti-de Sitter spacetime," Phys. Rev. D 59, 046003 (1999) [arXiv:hep-th/9805171].

[13] T. Banks, M. R. Douglas, G. T. Horowitz and E. J. Martinec, "AdS dynamics from conformal field theory," arXiv:hep-th/9808016.

[14] K. Behrndt, "About a class of exact string backgrounds," Nucl. Phys. B 455, 188 (1995) [arXiv:hep-th/9506106].

[15] R. Kallosh and A. D. Linde, "Exact supersymmetric massive and massless white holes," Phys. Rev. D 52, 7137 (1995) [arXiv:hep-th/9507022].

[16] M. Cvetic and D. Youm, "Singular BPS saturated states and enhanced symmetries of four-dimensional N=4 supersymmetric string vacua," Phys. Lett. B 359, 87 (1995) [arXiv:hep-th/9507160].

[17] S. J. Rey and J. T. Yee, "Macroscopic strings as heavy quarks in large N gauge theory and anti-de Sitter supergravity," Eur. Phys. J. C 22, 379 (2001) [arXiv:hep-th/9803001].

[18] J. M. Maldacena, "Wilson loops in large N field theories," Phys. Rev. Lett. 80, 4859 (1998) [arXiv:hep-th/9803002].

[19] A. W. Peet and J. Polchinski, "UV/IR relations in AdS dynamics," Phys. Rev. D 59, 065011 (1999) [arXiv:hep-th/9809022].

[20] S. Das, J. Gegenberg and V. Husain, "Scalar field spacetimes and the AdS/CFT conjecture," Phys. Rev. D 64, 065027 (2001) [arXiv:hep-th/0101169]. 
[21] V. Balasubramanian and P. Kraus, "A stress tensor for anti-de Sitter gravity," Commun. Math. Phys. 208, 413 (1999) [arXiv:hep-th/9902121].

[22] R. Emparan, C. V. Johnson and R. C. Myers, "Surface terms as counterterms in the AdS/CFT correspondence," Phys. Rev. D 60, 104001 (1999) [arXiv:hep-th/9903238].

[23] S. Hellerman, N. Kaloper and L. Susskind, "String theory and quintessence," JHEP 0106, 003 (2001) [arXiv:hep-th/0104180].

[24] E. Witten, "Anti-de Sitter space, thermal phase transition, and confinement in gauge theories," Adv. Theor. Math. Phys. 2, 505 (1998) [arXiv:hep-th/9803131].

[25] S. S. Gubser, "Curvature singularities: The good, the bad, and the naked," Adv. Theor. Math. Phys. 4, 679 (2002) [arXiv:hep-th/0002160].

[26] N. Drukker, D. J. Gross and N. Itzhaki, "Sphalerons, merons and unstable branes in AdS," Phys. Rev. D 62, 086007 (2000) [arXiv:hep-th/0004131].

[27] J. A. Harvey, P. Horava and P. Kraus, "D-sphalerons and the topology of string configuration space," JHEP 0003, 021 (2000) [arXiv:hep-th/0001143].

[28] A. Sen, "Rolling tachyon," JHEP 0204 (2002) 048 [arXiv:hep-th/0203211].

[29] A. Sen, "Tachyon matter," JHEP 0207, 065 (2002) [arXiv:hep-th/0203265].

[30] N. Lambert, H. Liu and J. Maldacena, "Closed strings from decaying D-branes," arXiv:hep-th/0303139.

[31] A. Buchel, P. Langfelder and J. Walcher, Annals Phys. 302 (2002) 78 [arXiv:hep-th/0207235].

[32] F. Leblond and A. W. Peet, "SD-brane gravity fields and rolling tachyons," JHEP 0304 (2003) 048 [arXiv:hep-th/0303035].

[33] M. Gutperle and A. Strominger, "Spacelike branes," JHEP 0204, 018 (2002) [arXiv:hep-th/0202210].

[34] C. M. Chen, D. V. Gal'tsov and M. Gutperle, "S-brane solutions in supergravity theories," Phys. Rev. D 66, 024043 (2002) [arXiv:hep-th/0204071].

[35] M. Kruczenski, R. C. Myers and A. W. Peet, "Supergravity S-branes," JHEP 0205, 039 (2002) [arXiv:hep-th/0204144].

[36] N. Ohta, "Intersection rules for S-branes," Phys. Lett. B 558, 213 (2003) [arXiv:hep-th/0301095].

[37] M. W. Choptuik, "Universality And Scaling In Gravitational Collapse Of A Massless Scalar Field," Phys. Rev. Lett. 70 (1993) 9.

[38] F. Pretorius and M. W. Choptuik, "Gravitational collapse in 2+1 dimensional AdS spacetime," Phys. Rev. D 62 (2000) 124012 [arXiv:gr-qc/0007008].

[39] V. Husain, G. Kunstatter, B. Preston and M. Birukou, "Anti-deSitter gravitational collapse," Class. Quant. Grav. 20 (2003) L23 [arXiv:gr-qc/0210011]. 